Revue d'Études Françaises

$\mathrm{N}^{\mathrm{o}} 24(2020)$

DOI : $10.37587 /$ ref.2020.1.18

\title{
MÁTÉ KovÁCS
}

\section{Alicja Kacprzak, Radka Mudrochová, Jean-François Sablayrolles (sous la dir. de), L'emprunt en question(s). Conceptions, réceptions, traitements lexicographiques, Limoges, Lambert-Lucas, 2019, 198.}

"Que voilà, me dira-t-on peut-être, un sujet rebattu! Un regard, en effet, sur la bibliographie placée en appendice et l'on verra qu'il n'est guère de langues dont on n'ait cherché à dénombrer les éléments empruntés, guère d'aspects de l'emprunt qui n'aient été ci ou là décelés et mis en évidence » (Duroy, 1956: 1). C'est avec ces quelques lignes que commence l'introduction du livre de Louis Duroy sur l'emprunt linguistique. Et nous sommes en 1956. La question se pose alors de savoir pourquoi faire paraître un volume sur la problématique de l'emprunt en 2019. Le livre faisant l'objet de ce compte rendu ne tarde pas à dissiper nos doutes éventuels en précisant, dès le début, qu'il s'agit d' « une approche du phénomène de l'emprunt plus distancée » qui se concentre sur "plusieurs de ces aspects théoriques posés par [c]e phénomène linguistique » (Kacprzak, Mudrochová, Sablayrolles, 2019 : 9).

Le présent ouvrage est le fruit d'une collaboration européenne entre plusieurs universités, les trois éditeurs représentant trois pays différents : Pologne (Alicja Kacprzak), République tchèque (Radka Mudrochová) et France (Jean-François Sablayrolles). Quant à sa structure, le volume est divisé en trois parties, ces dernières s'articulant, de manière logique et cohérente, autour des trois notions indiquées dans le sous-titre: conceptions, réceptions et traitements lexicographiques.

La première partie («Emprunt : concepts connexes et typologie ») - la plus volumineuse, comprenant au total cinq articles ayant pour sujet d'analyse trois langues différentes: le français, le polonais et le tchèque - traite essentiellement des questions théoriques liées à la classification et aux notions voisines de l'emprunt. La contribution inaugurant l'ouvrage, celle de JeanFrançois Sablayrolles, penche sur l'analyse minutieuse de diverses conceptions du phénomène de l'emprunt et de termes étroitement associés au contact des 
langues tels xénisme, pérégrinisme et statalisme, tout en puisant dans un grand éventail de ressources : articles, dictionnaires et encyclopédies. L'étude de John Humbley s'inscrit logiquement dans la continuité de l'article précédent et propose une réflexion approfondie sur la notion de xénisme. L'auteur parcourt la trajectoire de ce terme en linguistique française, en traductologie et dans les langues de spécialité, en particulier dans le domaine des finances, et s'interroge à la fin sur la pertinence du xénisme pour les études de néologie. Le troisième article de ce volet, rédigé par Alicja Kacprzak, met en lumière la perception de l'emprunt par les linguistes polonais. L'auteure se concentre sur l'analyse de deux points de vue jugés prépondérants en linguistique polonaise (emprunt comme phénomène langagier quasi-universel et comme résultat des influences subies par la langue polonaise au fil de l'histoire) pour terminer ensuite par la présentation de diverses typologies de l'emprunt en polonais. La contribution suivante, celle d'Anna Bobińska et d'Andrzej Napieralski, peut être considérée en quelque sorte comme le pendant de l'article précédent et traite la problématique de quelques approches méthodologiques préconisées par des linguistes polonais en ce qui concerne l'emprunt au français. Il est question essentiellement de la brève description des travaux de Bogdan Walczek et d'Anna Bochnakowa. L'étude clôturant cette partie de l'ouvrage a été écrite par Radka Mudrochová. À l'aide de dictionnaires traditionnels et en ligne ainsi que d'ouvrages linguistiques, l'auteure passe en revue la terminologie liée à l'emprunt en tchèque, présente des modèles de classification et tente de déterminer la place de l'emprunt au sein de la néologie.

La deuxième partie, intitulée "Réception des emprunts", rassemble trois articles destinés à examiner la question de l'accueil des emprunts du point de vue diachronique. Dans son étude, Giovanni Tallarico se livre à l'analyse des gallicismes dans la langue italienne en proposant un parcours du XVIII ${ }^{\mathrm{e}}$ au $\mathrm{XX}^{\mathrm{e}}$ siècle. L'auteur retrace ces différentes époques en soulignant les facteurs socioculturels et idéologiques qui ont exercé une influence considérable sur l'emprunt au français: gallomanie $\left(\mathrm{XVIII}^{\mathrm{e}}\right.$ siècle), purisme et idéaux démocratiques $\left(\mathrm{XIX}^{\mathrm{e}}\right.$ siècle) et nationalisme $\left(\mathrm{XX}^{\mathrm{e}}\right.$ siècle). Matthieu Pierens continue le panorama des langues par le néerlandais. Après avoir proposé une brève présentation de cette langue, l'auteur effectue un examen détaillé des emprunts en néerlandais selon les langues, en particulier les emprunts au français, au latin, à l'anglais et à l'allemand, esquisse l'attitude des locuteurs face à ce phénomène langagier et décrit le point de vue de la linguistique 
néerlandaise sur les emprunts. Dans le dernier article de cette partie, Jan Lazar aborde la thématique de la perception de l'emprunt dans le milieu linguistique tchèque. L'auteur propose un regard d'analyse sur les trois principales langues qui ont servi de source d'emprunt pour le tchèque : le français, l'anglais et l'allemand, en précisant pour chacune de ces langues le contexte historique, les champs sémantiques des mots empruntés ainsi que le degré d'intégration de ces derniers dans la langue tchèque.

Enfin, la troisième et dernière partie de l'ouvrage portant le titre « Emprunts, lexicographes et veille néologique » réunit deux contributions dans le domaine lexicographique, celles d'Anna Bochnakowa et d'Emmanuel Cartier. La première des deux études, rédigée par Anna Bochnakowa, présente l'attitude des lexicographes polonais face à l'emprunt $\mathrm{du} \mathrm{XIX}^{\mathrm{e}}$ au $\mathrm{XXI}^{\mathrm{e}}$ siècle. L'auteure examine de près un grand nombre de dictonnaires généraux et ceux spécialisés dans le traitement des mots d'origine étrangère, en accordant une attention spéciale à un projet récent en ligne : l'Observatoire linguistique de l'Université de Varsovie. Le dernier article du volume, celui d'Emmanuel Cartier, analyse les emprunts en français actuel à travers la présentation de la plateforme Néoveille. Après avoir rappelé quelques notions théoriques, l'auteur passe à la partie méthodologique de son étude, décrit les emprunts du point de vue linguistique, socio-pragmatique et diachronique pour arriver à la fin à l'examen des emprunts repérés et validés sur la plateforme Néoveille entre 2015 et 2017.

En conclusion, il ne nous reste qu'à recommander cet ouvrage collectif particulièrement riche en contenu à quiconque s'intéressant à la question de l'emprunt, ce "processus dynamique au cœur des échanges entre langues en contact » (Mejri, 2018: 107), et, en particulier, à tous ceux effectuant des recherches dans ce domaine.

\title{
Bibliographie
}

DUROY Louis (1956), L'Emprunt linguistique, Liège, Presses universitaires de Liège. MEJRI Salah (2018), "La part autochtone dans l'emprunt linguistique », Le français en Afrique, $\mathrm{n}^{\circ}$ 32, p. 89-109.

\author{
MÁTÉ KOVÁCS \\ Université Eötvös Loránd de Budapest \\ Courriel : kovacs.mate@btk.elte.hu
}

